\title{
Competencias digitales docentes y el reto de la educación virtual derivado de la covid-19
}

\section{Digital teaching skills and the challenge posed by virtual education as a result of Covid-19}

\author{
Josnel Martínez-Garcés \\ Jacqueline Garcés-Fuenmayor \\ Corporación Universitaria Autónoma de Nariño, Santiago de Cali, Colombia
}

Open Access:

ISSN: $0124-2121$

E-ISSN: $2665-2420$

ARTÍ́CULO RESULTADO DE INVESTIGACIÓN Copyright $\odot$ By Educación y Humanismo

Editor: Dhayana Fernández Matos Universidad Simón Bolívar

Correspondencia: Josnel Martínez martinezjosnel@gmail.com

Recibido: 24-04-2020 Aceptado: 20-06-2020 En línea desde: 07-07-2020 educativo que se presta en la institución.

Palabras clave: competencias digitales, COVID-19, educación superior, educación virtual.

\begin{abstract}
Objective: to determine the teachers' digital skills in relation to the implementation of virtual education in higher education in the department of Valle del Cauca, Colombia, as a consequence of the COVID-19 pandemic. Method: descriptive; the sample population was made up of 52 teachers working during the 2020 -I academic period. The sampling error was of $6.5 \%$. Data was gathered through two questionnaires with a reliability of 0.937 for both, and was measured through Cronbach's Alpha reliability coefficient. Results: the study highlights the ease to classify digital information $(78.85 \%)$, share information through virtual means $(50.00 \%)$, edit digital content $(40.38 \%)$, protect personal data $(40.38 \%)$, and develop conceptual skills $(42.31 \%)$. There are direct, positive, and strong correlations between three of the skills. The level of ability is placed in integrator II $(52.00 \%)$.. Discussion and Conclusions: informatization and information literacy, communication and cooperation, as well as problem-solving, are the most developed skills, while digital content creation and safety are the weakest ones. Few teachers reach an innovative level in terms of skills. Strengthening strategies should be implemented in case the mandatory quarantine resulting from the pandemic extends, and if quality in the educational service offered in the institution needs to be ensured.
\end{abstract}

Keywords: digital skills, COVID-19, higher education, virtual education. 


\section{Introducción}

El 06 de marzo de 2020 el Ministerio de Salud de la República de Colombia (2020) anuncia el primer caso confirmado de COVID-19 en el país. Desde ese momento, se activan las alarmas de alerta en todo el territorio nacional donde de manera articulada el gabinete ministerial junto a las autoridades departamentales y municipales establecen estrategias de distanciamiento social que suponen la completa reorganización del sistema socioeconómico.

En este marco, el presidente de la República Dr. Iván Duque Márquez solicita que desde el lunes 16 de marzo de 2020 hasta el viernes 20 de marzo de 2020 las instituciones de educación superior desarrollen estrategias de educación virtual como medida alternativa de distanciamiento social; exigencia que tras el decreto 457 emitido por la Presidencia de la República (2020) se vuelve obligatoria hasta el 13 de abril de 2020, extendida hasta el 27 de abril de 2020 (El Tiempo, 2020) y prorrogable, al menos, hasta el 11 de mayo de 2020 (Semana, 2020).

Esta situación representa facilidad de trabajo para algunos docentes, pero serias dificultades para otros que, tal como afirman Martínez, Burbano y Burbano (2019), consideran el uso de TIC como una dificultad en su proceso de enseñanza. Sin embargo, la educación virtual no es algo nuevo y su implementación en las instituciones de educación superior ha sido progresiva.

Fernández, Govea y Belloso (2002) afirman que las primeras representaciones de esta modalidad tenían un esquema semipresencial - también llamado educación a distancia - cuyo propósito era poder ampliar la oferta a los estudiantes con unos costos más bajos brindándoles así la alternativa de matricular asignaturas adicionales de manera rápida y económica. Esta opción resulta especialmente factible, aunque no única, para el desarrollo de las propuestas curriculares en las instituciones formativas de administración pública que suelen tener una alta precariedad de recursos financieros y educativos (Olmedo, Gómez \& Pintor, 2014).

Copertari y Sgreccia (2011) sostienen que la implementación de esta modalidad de estudio no presencial permite: a) un estilo de gestión académica al servicio de los estudiantes, b) aprendizaje colaborativo en red a través de los recursos pedagógicos y didácticos disponibles, c) el desarrollo de habilidades cognitivas y metacognitivas en los estudiantes que les ayudan a alcanzar una comprensión genuina orientada mediante acciones tutoriales, d) un sistema de evaluación procesual y permanente igual al de modalidades presenciales, e) flexibilización de la accesibilidad, permanencia y egreso de los estudiantes y f) recursos tecnológicos y didácticos que promueven la interactividad. 
Además de lo anterior, Varguillas y Bravo (2020) enuncian entre las bondades de la modalidad de aprendizaje virtual: a) la abundancia de información web disponible en diferentes formatos, b) el uso de recursos tecnológicos adicionales a los de una clase presencial, como foros, chats y videoconferencias, c) la posibilidad de un aprendizaje más interactivo y participativo, d) desarrollo de la autonomía estudiantil y e) la retroalimentación asincrónica para aprovechar mejor el tiempo de los estudiantes.

Así, estos espacios de aprendizaje virtual resultan altamente provechosos para aquellos estudiantes que por su trabajo u ocupaciones diversas no pueden tener la opción de ingresar a un sistema de formación presencial, siendo estos entonces una alternativa ideal para el desarrollo de sus actividades académicas y la consecución de sus metas profesionales (Andrade y Andrade, 2017). Además, la adquisición de competencias digitales en el proceso de enseñanza-aprendizaje permite hacer frente a los cambios sociales, y resultan eficaces al momento de brindar una respuesta a las necesidades de una sociedad cada día más globalizada (Martínez y Lugo, 2018; Martínez, 2017; García y Vírseda, 2016; Duque, 2016).

Sin embargo, desde el punto de vista docente, el uso de las herramientas de comunicación e información en ambientes de formación virtual es limitado, condicionado a la motivación y en muchos casos débiles en cuanto a su formación para manejarlas; muchos carecen de la experticia suficiente en el uso de instrumentos que puedan ser empleados en su didáctica y metódica no presencial (Pincay, 2018; Cabero y Marín, 2014). Para Osuna y López (2015) esta situación se vuelve crítica cuando se tiene presente que la información y la comunicación no son capaces de generar conocimiento por sí mismas a menos que sean mediadas con estrategias que contribuyan al desarrollo de un pensamiento crítico en los participantes.

Por este contexto de pandemia mundial generada por la COVID-19 y su consecuente distanciamiento social donde no hay otra opción distinta que adaptarse a la educación virtual para darle consecución a los procesos educativos, toma especial relevancia el hecho de que los docentes tengan competencias digitales para el manejo de recursos que sean útiles en sus clases y de provecho para los estudiantes. De esta manera surge la presente investigación donde se pretende determinar las competencias digitales de los docentes para poder hacer frente a la implementación de la educación virtual en una institución de educación superior ubicada en el departamento Valle del Cauca Colombia como consecuencia de la pandemia generada por la COVID-19.

Se conceptualizan las competencias digitales del docente como su habilidad para la comprensión, uso y evaluación crítica de medios digitales de comunicación (Ala-Mutka, Punie \& Redecker, 2008). Krumsvik (2008) considera además que el uso de estas herramientas para el proceso de enseñanza-aprendizaje debe fundamentarse en criterios didácticos y pedagógicos con conciencia ética y moral. Así, una competencia digital del 
docente implica la relación entre disciplina, pedagogía y tecnología (Koehler \& Mishrsa, 2009). Se considera entonces el Marco común de competencia digital docente publicado a través del Instituto Nacional de Tecnologías Educativas y de Formación del Profesorado (2012) donde se establece que las competencias digitales que todo docente debe desarrollar son: 1) informatización y alfabetización informacional, 2) comunicación y elaboración, 3) creación de contenido digital, 4) seguridad y 5) resolución de problemas.

\section{Método}

Los materiales y métodos utilizados en la investigación se detallan a continuación:

La metodología empleada es de tipo descriptiva, fundamentada en la descripción, registro y análisis del objeto estudiado en su ambiente natural (Tamayo, 2004). Los datos fueron recolectados directamente del escenario real donde se desarrollaron y sin someter la variable estudiada a algún tipo de manipulación (Hurtado, 2010; Arias, 2004). En cuanto al período de tiempo abarcado se considera un estudio transaccional pues la medición se hizo en un único momento en el tiempo, específicamente el 16 de abril de 2020, cuando se cumplió un mes de la transición obligatoria a la modalidad de educación virtual en la institución estudiada como consecuencia del aislamiento social ocasionado por la pandemia de la COVID-19 (Hernández, Fernández y Batista, 2006).

\section{Participantes}

En concordancia con el objetivo planteado, los sujetos participantes de la investigación fueron los miembros del personal docente y del personal administrativo con descarga docente vinculados a la institución de educación superior objeto de análisis durante el periodo académico 2020-I, momento en el cual inicia la propagación de la pandemia de la COVID-19 en Colombia. Los mismos ascienden a un total de sesenta y siete (67) docentes a los cuales se les suministró un cuestionario vía Google forms para ser respondido de manera voluntaria. Se recolectaron cincuenta y dos (52) encuestas diligenciadas obteniendo así un error muestral de 6,5\% y demostrando la alta representatividad de la muestra frente al total de la población.

\section{Instrumentos}

La técnica de recolección de datos empleada fue la encuesta y los instrumentos diseñados para ello fueron dos cuestionarios. El primero se orientó a la descripción de competencias digitales y estuvo integrado por quince (15) ítems con opciones de respuesta en escala Likert con opciones de respuesta del 1 al 4 donde 1 corresponde a "Difícil" y 4 corresponde a "Fácil" con puntos intermedios de tendencia positiva y negativa, el segundo se enfocó en determinar el nivel de competencias digitales a través de ocho (8) ítems con opciones de respuesta dicotómica; ambos en correspondencia a las dimensiones establecidas para la variable de investigación "competencias digitales del docente" (ver Tabla 1: operacionalización de la variable). La confiabilidad se determinó calculando el coeficiente Alpha-Cronbach el cual, con $100 \%$ de casos validos frente a las 
cincuenta y dos (52) observaciones, arrojó un resultado de 0,937 para ambos instrumentos demostrando que estadísticamente son confiables y de estructura robusta.

Tabla 1

Operacionalización de la variable

\begin{tabular}{ccc}
\hline Variable & Dimensión & Ítems \\
\hline \multirow{3}{*}{$\begin{array}{c}\text { Competencias } \\
\text { digitales del docente }\end{array}$} & Informatización y alfabetización informacional & $1,2,3$ \\
\cline { 2 - 3 } & Comunicación y colaboración & $4,5,6$ \\
\cline { 2 - 3 } & Creación de contenido digital & $7,8,9$ \\
\cline { 2 - 3 } & Seguridad & $10,11,12$ \\
\cline { 2 - 3 } & Resolución de problemas & $13,14,15$ \\
\hline
\end{tabular}

Fuente: elaboración propia (2020).

Para la presentación de los resultados y su discusión se elaboraron tablas con estadística descriptiva expresando cifras en frecuencia relativa. Asimismo, para el análisis de correlaciones se calculó el coeficiente Rho de Spearman ( $\rho$ ) utilizando como herramienta de apoyo el Statistical Package for the Social Sciences (SPSS) v.26.0.

\section{Resultados y discusión}

Para Moll (2018), la primera competencia digital docente es la informatización y alfabetización informacional. Esta consiste en la facilidad para localizar, identificar y clasificar la información digital considerando su finalidad y relevancia. En este sentido, Tarango y Machado (2012) sostienen que a través de esta competencia un individuo es capaz de hacer uso de los conocimientos acumulados y del acervo de experiencias de otros profesionales para, a través de las tecnologías de comunicación e información, nutrir a nuevas generaciones con un enfoque hacia la cibercultura. En la Tabla 2 se muestras los resultados obtenidos sobre esta competencia en el caso universitario estudiado.

Tabla 2

\begin{tabular}{ccccc}
\multicolumn{5}{c}{ Informatización y alfabetización informacional } \\
\hline Localizar & $\mathbf{1}$ & $\mathbf{2}$ & $\mathbf{3}$ & $\mathbf{4}$ \\
\hline Identificar & $5,77 \%$ & $5,77 \%$ & $30,77 \%$ & $57,69 \%$ \\
\hline Clasificar & $3,85 \%$ & $13,46 \%$ & $25,00 \%$ & $57,69 \%$ \\
\hline & $3,85 \%$ & $1,92 \%$ & $15,38 \%$ & $78,85 \%$ \\
\hline & Difícil & $<$ & $>$ & Fácil \\
\hline
\end{tabular}

Fuente: elaboración propia (2020).

En la Tabla 2 se observa cómo entre los sujetos participantes de la investigación un $57,69 \%$ consideran que para ellos es fácil localizar en la red contenido digital relevante para sus clases, demostrando de esta manera que a través de bibliotecas y repositorios 
digitales satisfacen sus necesidades de información junto a un 30,77\% que también mantiene una tendencia positiva en este sentido (Caraballo y Castro, 2015).

De igual manera, un $57,69 \%$ considera fácil y un $25 \%$ tiene una tendencia positiva a identificar cuál del contenido localizado es más idóneo para sus clases, lo cual contribuye a que el uso y evaluación de la información utilizada sea útil para la generación de nuevos conocimientos (Cruz, 2009). Sin embargo, resalta el hecho de que un 13,46\% respondió negativamente a este indicador evidenciando que se las hace difícil esta acción. En cuanto a clasificar la información, un 78,85\% lo considera fácil y 15,38\% mantiene una tendencia positiva sobre ello. La adecuada clasificación es lo que facilita la ubicación del material digital (bien sea en un almacenamiento local o en la web) para su posterior consulta (Delis, 2005).

La segunda competencia digital docente es la comunicación y colaboración. Esta es la que permite a los docentes utilizar entornos digitales para compartir recursos utilizando herramientas en línea promoviendo la conexión y colaboración con otros a través de la construcción de redes de socialización (Moll, 2018). Al respecto, la Tabla 3 muestra los resultados obtenidos entre los sujetos abordados.

Tabla 3

Comunicación y colaboración

\begin{tabular}{lcccc}
\hline Comunicar & $3,85 \%$ & $13,46 \%$ & $48,08 \%$ & $34,62 \%$ \\
\hline Compartir & $1,92 \%$ & $9,62 \%$ & $38,46 \%$ & $50,00 \%$ \\
\hline Colaborar & $5,77 \%$ & $21,15 \%$ & $44,23 \%$ & $28,85 \%$ \\
\hline & Difícil & $<$ & $>$ & Fácil \\
\hline
\end{tabular}

Fuente: elaboración propia (2020).

Se aprecia en la Tabla 3 que existe una tendencia medianamente positiva de $48,08 \%$ en cuanto a la facilidad para comunicar, sin embargo, una cantidad significativa de $34,62 \%$ consideran que establecer comunicación con sus estudiantes bajo entornos digitales es definitivamente fácil contemplando el uso adecuado de los distintos formatos disponibles (Cantón, Cañón y Grande, 2017).

En cuanto a compartir, la tendencia se mantiene positiva donde un 50,00\% afirma que para ellos es fácil, junto a un 38,46\% que también se alinea a ese criterio, compartir contenido con sus estudiantes en entornos digitales alentando la conexión y la colaboración (Arroyo, 2017). Sobre este último aspecto, colaborar presenta una tendencia medianamente positiva de $48,08 \%$ en cuanto a facilidad, sin embargo, una cantidad significativa de $34,62 \%$ consideran que colaborar con otros docentes para la formación de sus estudiantes en entornos digitales es definitivamente fácil promoviendo así el intercambio de ideas y las relaciones interpersonales entre docentes y alumnos (Castro, Cedillo y Valenzuela, 2015). 
La tercera competencia digital docente es la creación de contenido digital. En ella juega un papel especial la creatividad para poder generar, editar, integrar y reelaborar contenidos digitales teniendo presente los aspectos de la propiedad intelectual y las licencias de uso como creative commons (Moll, 2018). En la Tabla 4 se recogen los resultados obtenidos sobre esta competencia digital en los docentes consultados.

Tabla 4

Creación de contenido digital

\begin{tabular}{ccccc}
\hline Crear & $1,92 \%$ & $23,08 \%$ & $42,31 \%$ & $32,69 \%$ \\
\hline Editar & $3,85 \%$ & $17,31 \%$ & $38,46 \%$ & $40,38 \%$ \\
\hline Manejar (licencias) & $23,08 \%$ & $36,54 \%$ & $19,23 \%$ & $21,15 \%$ \\
\hline & Difícil & $<$ & $>$ & Fácil \\
\hline
\end{tabular}

Fuente: elaboración propia (2020).

En cuanto a crear, la tendencia se mantiene positiva donde un 32,69\% afirma que para ellos es fácil, junto a un $42,31 \%$ que también se alinea a ese criterio, producir contenido digital completamente nuevo para utilizarlo en sus clases bajo entornos digitales. De esta manera se producen contenidos y programas nuevos para su uso en la práctica educativa (Vargas, 2019).

Editar presenta una tendencia positiva de $38,46 \%$ sumado a un $40,38 \%$ que aseguran es fácil para ellos la edición de contenido previamente existente y adaptarlo a las necesidades de sus clases bajo entornos virtuales. Un 17,31\% muestra una inclinación negativa ante este aspecto. En este sentido, la realización de cambios en material digital debe respetar las restricciones de uso y la debida citación (Martínez \& Rodríguez, 2018). Sobre este aspecto, el ítem manejar licencias como creative commons presenta una notable tendencia negativa de $36,54 \%$ y de un $23,08 \%$ que considera definitivamente difícil identificar y manejar estos permisos de uso posiblemente debido a una falta de cultura ante el manejo de derechos de autor (Castillejos, 2007).

La cuarta competencia es la de seguridad relacionada con los conocimientos, actitudes y habilidades de los docentes para diseñar y desarrollar experiencias de aprendizaje digitalmente responsables contando con la adecuada privacidad, integridad y eficiencia de la información manejada a través de internet (Gallego, Torres y Pessoa, 2019). Por ello, para Torres, Pessoa y Gallego (2019) la seguridad digital y la disminución de sus problemas asociados va de la mano con tomar conciencia sobre los riesgos que existen en el mundo virtual. A continuación, la Tabla 5 expone los resultados obtenidos con relación a esta competencia.

Tabla 5

Seguridad

\begin{tabular}{ccccc}
\hline Seguridad informática & $15,38 \%$ & $30,77 \%$ & $32,69 \%$ & $21,15 \%$ \\
\hline Protección de datos & $19,23 \%$ & $25,00 \%$ & $40,38 \%$ & $15,38 \%$ \\
\hline
\end{tabular}




\begin{tabular}{ccccc} 
Protección de identidad & $17,31 \%$ & $32,69 \%$ & $30,77 \%$ & $19,23 \%$ \\
\hline & Difícil & $<$ & $>$ & Fácil \\
\hline \multicolumn{6}{c}{ Fuente: elaboración propia (2020). }
\end{tabular}

En cuanto a hacer uso de seguridad informática al momento de desarrollar sus clases bajo entornos digitales las respuestas se concentran en tendencias no absolutas donde, un $32,69 \%$ se inclina a la facilidad mientras que un $30,77 \%$ considera que por el contrario suele ser difícil utilizarla. Por otra parte, la protección de datos personales muestra una alta tendencia positiva $(40,38 \%)$ a que es fácil para los docentes encuestados mantenerla. Sin embargo, la protección de identidad si se mantiene con una inclinación negativa de 32,69\% y un 17,31\% que consideran definitivamente difícil establecer protocolos de protección digital al momento de desarrollar sus clases bajo entornos virtuales. En este sentido, la protección y seguridad son muy importantes y se debe considerar como una oportunidad para alcanzar la alfabetización digital (Arrieta y Montes, 2011).

La quinta y última competencia es la resolución de problemas, orientada a la identificación de necesidades, toma de decisiones y resolución de problemas conceptuales a través de herramientas digitales usando de manera creativa la tecnología para actualizar las competencias propias y colectivas (Moll, 2018). Seguidamente, la Tabla 6 recoge los resultados obtenidos sobre esta competencia.

Tabla 6

Resolución de problemas

\begin{tabular}{ccccc}
\hline Resolver & $7,69 \%$ & $11,54 \%$ & $46,15 \%$ & $34,62 \%$ \\
\hline Usar & $3,85 \%$ & $17,30 \%$ & $36,54 \%$ & $42,31 \%$ \\
\hline Desarrollar & $7,69 \%$ & $11,54 \%$ & $38,46 \%$ & $42,31 \%$ \\
\hline & Difícil & $<$ & $>$ & Fácil \\
\hline
\end{tabular}

Fuente: elaboración propia (2020).

Se observa en la Tabla 6 que resolver competencias conceptuales sobre sus asignaturas dictadas bajo entornos digitales concentra una tendencia positiva de $46,15 \%$ junto a un $34,62 \%$ que considera para ellos es absolutamente fácil, orientando así la construcción del conocimiento desde la virtualidad (Durán, Gutiérrez y Paz, 2016). Asimismo, en cuanto a usar de manera creativa la tecnología en sus asignaturas dictadas bajo entornos digitales los resultados se concentran altamente positivos con un $36,54 \%$ sumado a un $42,31 \%$ que lo considera absolutamente fácil. Para Marcelo (2013), esta creatividad es la que permite encontrar la manera más óptima de atender las dificultades de aprendizaje en los estudiantes y redirigir sus problemas.

En este orden de ideas, un $38,46 \%$ muestra una tendencia positiva junto a un $42,31 \%$ que considera completamente fácil desarrollar competencias digitales propias en sus 
asignaturas dictadas bajo entornos virtuales permitiendo así una apropiación tecnológica desde su área de conocimiento, mediante recursos formativos, para orientar de manera correcta los aprendizajes y habilidades de los estudiantes (Albertos, Domingo y Albertos, 2016).

Siguiendo este orden lógico, se calcularon las correlaciones entre las competencias digitales docentes de la población estudiada; para ello, se asignaron nomenclaturas a cada una de ellas donde: información y alfabetización informacional = IAIX, comunicación y colaboración $=\mathrm{CEX}$, creación de contenido digital $=\mathrm{CCDX}$, seguridad $=\mathrm{SX}$ y resolución de problemas $=$ RPX. Así las cosas, los resultados obtenidos al respecto se muestran en la Tabla 7.

Tabla 7

Correlaciones de las competencias digitales docentes (Rho de Spearmanp)

\begin{tabular}{|c|c|c|c|c|c|c|}
\hline & & IAIX & CEX & CCDX & sX & RPX \\
\hline & Coeficiente de correlación & 1,000 &, $620^{* *}$ &, $702^{* *}$ & $471^{* *}$ & $703^{* *}$ \\
\hline \multirow[t]{3}{*}{ IAIX } & Sig. (bilateral) & &, 000 &, 000 &, 000 & 000 \\
\hline & $\mathrm{N}$ & 52 & 52 & 52 & 52 & 52 \\
\hline & Coeficiente de correlación & $620^{* *}$ & 1,000 &, $667^{* *}$ &, $513^{* *}$ &, $665^{* *}$ \\
\hline \multirow[t]{3}{*}{ CEX } & Sig. (bilateral) &, 000 & & ,000 &, 000 &, 000 \\
\hline & $\mathrm{N}$ & 52 & 52 & 52 & 52 & 52 \\
\hline & Coeficiente de correlación & ,702 &, $667^{* *}$ & 1,000 &, $577^{* *}$ & $710^{* *}$ \\
\hline \multirow[t]{3}{*}{ CCDX } & Sig. (bilateral) &, 000 &, 000 & &, 000 &, 000 \\
\hline & $\mathrm{N}$ & 52 & 52 & 52 & 52 & 52 \\
\hline & Coeficiente de correlación & $471^{* *}$ &, $513^{* *}$ &, $577^{* *}$ & 1,000 &, $539^{* *}$ \\
\hline \multirow[t]{3}{*}{ sX } & Sig. (bilateral) &, 000 &, 000 &, 000 & &, 000 \\
\hline & $\mathrm{N}$ & 52 & 52 & 52 & 52 & 52 \\
\hline & Coeficiente de correlación & $703^{* *}$ & $665^{* *}$ &, $710^{* *}$ &, $539^{* *}$ & 1,000 \\
\hline \multirow[t]{2}{*}{ RPX } & Sig. (bilateral) &, 000 &, 000 &, 000 &, 000 & \\
\hline & $\mathrm{N}$ & 52 & 52 & 52 & 52 & 52 \\
\hline
\end{tabular}

La Tabla 7 muestra que las principales correlaciones existentes son:1) Resolución de problemas-Informatización y alfabetización informacional $(\rho=, 703), 2)$ Informatización y alfabetización informacional-Creación de contenido digital $(\rho=, 702)$ y 3) Creación de contenido digital-Resolución de problemas $(\rho=710)$. Así, mientras más fácil sea para el docente localizar en la red contenido digital, identificar cuál de ese contenido es el más idóneo para sus clases, así como almacenarlo y clasificarlo para su posterior consulta, entonces podrá ser más efectivo en cuanto a resolver competencias conceptuales de su asignatura y usar la tecnología de manera creativa junto a lo relacionado con la creación y edición de material digital apegados al uso de licencias como Creative Commons. 
Finalmente, para determinar el nivel de competencia digital en los docentes encuestados se adaptó el modelo propuesto por el Ministerio de Educación Nacional (2013) estableciendo tres niveles con sus respectivos subniveles, a saber, exploración (I, II, III, IIII), integración (I, II, III) e innovación. En la gráfica 1 se recogen los resultados obtenidos con relación a esto.

Gráfica 1

Nivel de competencia digital

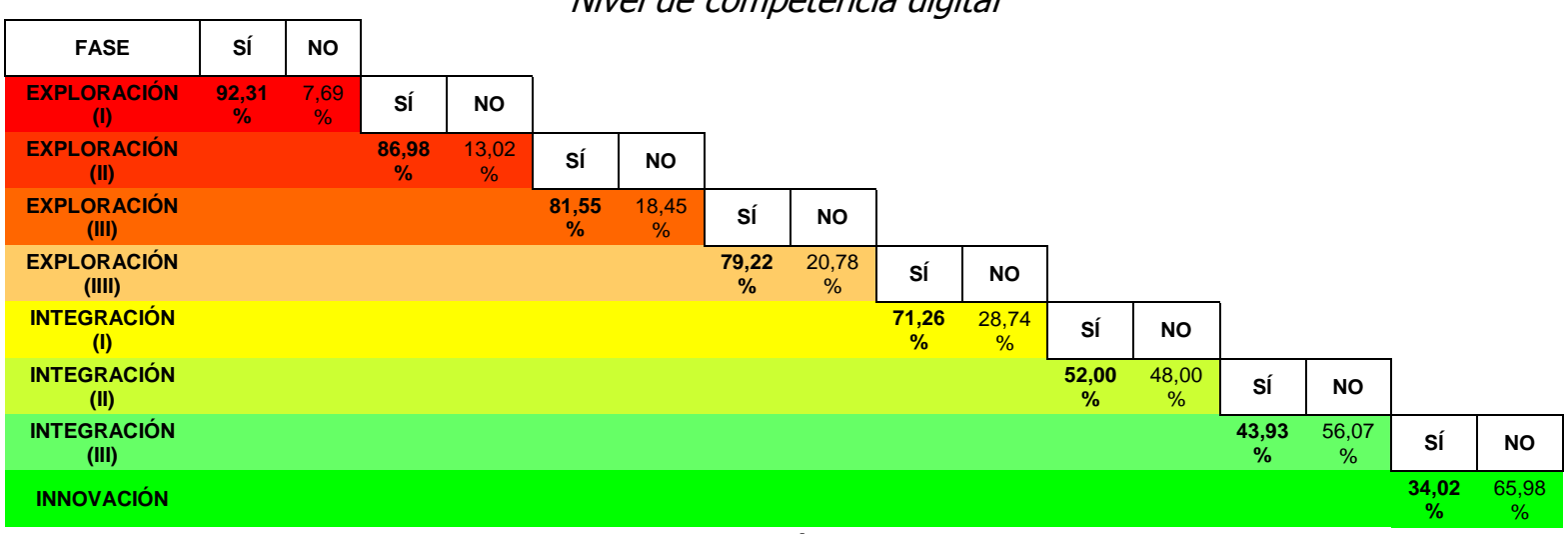

Fuente: elaboración propia (2020).

En la Gráfica 1 se puede observar que el nivel Integración IIes el que cubre a la mayor parte de los docentes encuestados $(52,00 \%)$ antes que la tendencia se vuelva reversible. En este nivel se considera que los docentes: 1) usan las TIC por sí mismos, 2) las utilizan en sus labores cotidianas, 3) entienden las implicaciones éticas de su uso en los procesos educativos, 4) las integran de manera pertinente con el Proyecto educativo institucional, 5) combinan distintas herramientas para el diseño de ambientes de aprendizaje y 6) se consideran pioneros en adoptar nuevas ideas tecnológicas. Resulta relevante que sólo un 34,02\% llega al nivel de Innovación donde, además de lo anterior, son capaces de 1) establecer criterios para argumentar cómo las TIC facilitan el aprendizaje y 2) compartir sus actividades aplicadas ajustarlas de acuerdo a la retroalimentación con sus compañeros.

\section{Conclusiones}

Sin duda, esta emergencia sanitaria generada por la COVID-19 ha causado estragos en todos los rincones del planeta y traerá repercusiones futuras que modificarán los estilos de vida y consumo. El sector educativo, como componente esencial de la sociedad, deberá adaptarse a estas nuevas demandas donde la virtualidad, que antes era una opción, ya se ha convertido en una modalidad prácticamente de carácter obligatorio. Por ello, el desarrollo y fortalecimiento de competencias digitales en los docentes resultan imperativos. Con relación a los encuestados que se constituyeron en sujetos de esta 
investigación, y atendiendo al objetivo planteado para la misma, se pudo determinar que entre las cinco competencias digitales esenciales para el desarrollo académico:

a. Informatización y alfabetización informacional. Tienen facilidad para localizar información digital a través de bibliotecas y repositorios en la red que sea útil para sus clases, identificando cuál es la más idóneo para la generación de nuevos conocimientos pudiéndolos clasificar adecuadamente para su posterior consulta, bien sea desde un almacenamiento local o desde la misma web.

b. Comunicación y colaboración. La comunicación con sus estudiantes a través de medios digitales es fluida y concebida a través de distintos formatos permitiéndoles de esta manera compartir contenidos y experiencias que fortalecen la conexión y la colaboración. Esta última se considera especialmente sencilla al momento de establecer relaciones personales y profesionales con sus pares académicos para la consecución de los objetivos formativos.

c. Creación de contenido digital. Es una de las competencias más débiles pues, aunque la mayoría considera fácil crear contenidos digitales para su práctica educativa, presentan dificultades al momento de editar material digital ya existente y muchas limitaciones en lo relacionado a la identificación y manejo de licencias de uso como Creative Commons, evidenciando una falta de cultura en cuanto al manejo de derechos de autor.

d. Seguridad. Es la competencia que se presenta más débil entre todas. Aspectos como protección de datos personales, protección de identidad y protección digital se manejan con dificultad lo que impide, además de garantizar la protección y seguridad del manejo de información en la red, alcanzar un crecimiento en cuanto a la alfabetización digital.

e. Resolución de problemas. Se considera altamente desarrollada, a través de ella los docentes consideran que con facilidad pueden resolver competencias digitales en torno a las asignaturas que dictan utilizando de manera creativa la tecnología y apropiándose de estas, generando recursos formativos que direccionen los conocimientos y habilidades de los alumnos.

En cuanto a las correlaciones, se concluye que existe una importante necesidad de desarrollar en el corto plazo un plan de fortalecimiento para las competencias relacionadas con la seguridad informática y la comunicación y elaboración de contenido digital previendo una extensión de las clases virtuales, por lo menos, hasta finales del año 2020. Esto debido a que fueron las que presentaron correlaciones más débiles y poco positivas.

Finalmente, en cuanto al nivel de competencia digital alcanzado, la mayoría de los 
docentes abordados se encuentran en la etapa de Integración II donde se considera que son capaces de hacer uso de tecnologías de información por sí mismos en su vida personal y laboral, articulándolas a los objetivos del Proyecto educativo institucional para el desarrollo de herramientas virtuales de aprendizaje tomando en consideración aspectos éticos y morales.

Sin embargo, son pocos los que alcanzan el nivel Innovador, situación que permite orientar las estrategias futuras para el fortalecimiento de sus competencias digitales, previendo que posiblemente el aislamiento preventivo obligatorio producto de la pandemia generada por la COVID-19 se extienda por un período de tiempo mayor, pero que de igual manera se debe garantizar la calidad del servicio educativo que se presta en la institución.

\section{Referencias}

Ala-Mutka, K., Punie, Y. \& Redecker, C. (2008). Digital competence for lifelong learning. Madrid, España: JRC EuropeanCommission.

Albertos, A., Domingo, A., y Albertos, J. (2016). Estrategia docente para el desarrollo de la competencia digital en el aula universitaria.: del uso recreativo al uso formativo. Educar, 52(2), 243-261. https://educar.uab.cat/article/viewFile/v52-n2-albertosdomingo-albertos/732-pdf-es

Andrade, L., y Andrade, M. (2017). Uso de las rúbricas en ambientes virtuales de

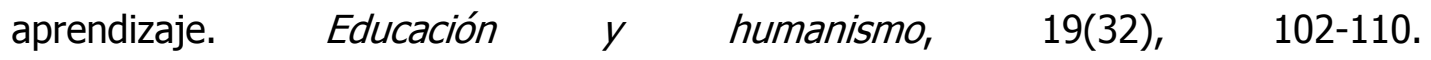
https://doi.org/10.17081/eduhum.19.32.2535

Arias, F. (2004). El proyecto de investigación. Introducción a la metodología científica. Caracas, Venezuela: Editorial Episteme.

Arrieta, A., y Montes, D. (2011). Alfabetización digital: uso de las TIC's más allá de una formación instrumental y una buena infraestructura. Revista colombiana de ciencia animal, 3(1), 180-197. https://revistas.unisucre.edu.co/index.php/recia/article/view/360

Arroyo, A. (2017). Competencias en comunicación y colaboración en la formación de docentes. Revista mediterránea de comunicación, 8(2), 277-285. https://rua.ua.es/dspace/bitstream/10045/67619/1/ReMedCom_08_02_20_esp.pdf

Cabero, J., y Marín, V. (2014). Miradas sobre la formación del profesorado en tecnologías de información y comunicación (TIC). Enl@ce. Revista venezolana de información, 
tecnología $\quad y \quad$ conocimiento, 11(2), 11-24. http://produccioncientificaluz.org/index. php/enlace/article/view/18866/18839

Cantón, I., Cañón, R., y Grande, M. (2017). La comunicación como subdimensión de la competencia digital en futuros maestros de primaria. Pixel-bit. Revista de medios y educación, (50), 33-47. https://www.redalyc.org/pdf/368/36849882002.pdf

Caraballo, K., y Castro, M. (2015). Programa de alfabetización informacional para el personal bibliotecario de la Universidad de Sancti Spíritus. Revista cubana de información en ciencias de la salud, 26(2), 168-186. http://scielo.sld.cu/pdf/ics/v26n2/rci06215.pdf

Castillejo, B. (2007). Gestión de información y creación de contenido digital en el prosumidormillennial. Apertura, 11(1), 24-39. http://dx.doi.org/10.32870/Ap.v11n1.1375

Castro, M., Cedillo, M., y Valenzuela, J. (2015). Apropiación de las competencias digitales mediante el uso de tabletas iPads en alumnos de sexto grado de primaria. Revista iberoamericana de educación, 68(2), 123-140. https://rieoei.org/historico/deloslectores/6913.pdf

Copertari, S., y Sgreccia, N. (2011). Postgrados a distancia y virtualización en la Universidad Nacional de Rosario. Educación y humanismo, 13(20), 14-32. http://revistas.unisimon.edu.co/index.php/educacion/article/view/2279

Cruz, I. (2009). Programa de alfabetización informacional para bibliotecarios [Tesis de maestría, Universidad de la Habana, Cuba]. Repositorio Institucional Flacsoandes. https://repositorio.flacsoandes.edu.ec/bitstream/10469/1367/1/TFLACSO2009IGCD.pdf

Delis, L. (2005). La alfabetización informacional en el contexto de la formación continua. [Tesis de maestría, Universidad de la Habana, Cuba]. Repositorio. https://www.aacademica.org/liliam.maria.delis.alfonso/5.pdf

Duque, E. (2016). Adquisición de competencias digitales para la inclusión social. Opción, 32(9), 610-630. http://produccioncientificaluz.org/index.php/opcion/article/view/21765/21539

Durán, M., Gutiérrez, I., y Paz, M. (2016). Análisis conceptual de modelos de competencia digital del profesorado universitario. Revista latinoamericana de tecnología educativa, 15(1), 97-114. http://dehesa.unex.es/bitstream/handle/10662/5790/1695- 


\section{X_15_1_97.pdf?sequence=1}

El Tiempo (07 de abril de 2020). Siete datos claves que debe saber sobre la extensión del aislamiento. https://www.eltiempo.com/politica/gobierno/claves-de-ampliacion-decuarentena-hasta-el-29-de-abril-2020-ordenada-por-ivan-duque-481782

Fernández, C., Govea, M., y Belloso, O. (2002). La universidad virtual en Venezuela. Un estudio de caso. Revista de Ciencias sociales, VIII (1), 170-180. http://produccioncientificaluz.org/index.php/rcs/article/view/25169

Gallego, M., Torres, N., y Pessoa, T. (2019). Competencia de futuros docentes en el área de seguridad digital. Comunicar, 61(XXVII), 57-67. https://doi.org/10.3916/C612019-05

García, F., \& Virseda, E. (2016). Inclusión de competencias digitales en los estudios de grado en Trabajo Social. Opción, 32(Especial 9), 802-820. http://produccioncientificaluz.org/index.php/opcion/article/view/21776/21550

Hernández, R., Fernández, C., y Batista, L. (2006). Metodología de investigación. Ciudad de México, México: Editorial McGraw Hill.

Hurtado, J. (2010). El proyecto de investigación. Caracas, Venezuela: Ediciones Quirón.

Instituto Nacional de Tecnologías Educativas y de Formación del Profesorado (2012). Marco común de competencia digital docente. Madrid, España: Ministerio de educación, cultura y deporte. http://eduteka.icesi.edu.co/pdfdir/intef-competenciadigital-docente-2017.pdf

Koehler, M. \& Mishra, P. (2009). What is technological pedagogical content knowledge? Contemporary issues in technology and teacher education, 9(1), 60-70. https://tecfalabs.unige.ch/mitic/articles/koehler_mishra_2009_what_is_technologic al_pedagogical_content_knowledge.pdf

Krumsvik, R. (2008). Situated learning and teachers' digital competence. Education and information technologies, 13(4), 279-290. https://link.springer.com/article/10.1007/s10639-008-9069-5\#page-1

Marcelo, C. (2013). Las tecnologías para la innovación y la práctica docente. Revista $\begin{array}{llll}\text { brasileira de educação, 25-47. } & \text { 18(52), }\end{array}$ http://www.scielo.br/pdf/rbedu/v18n52/03.pdf

Martínez, J. (2017). Desarrollo del capital humano para la construcción del desarrollo 
endógeno. En Mata, J.; Ruíz, J., y Atienzo, H. Memorias arbitradas 1era Jornada de divulgación y socialización científica, tecnológica e innovativa para el desarrollo endógeno en Venezuela (393-396). Cabimas, Venezuela: Universidad Nacional Experimental Rafael María Baralt.

Martínez, J., Burbano, M., y Burbano, E. (2019). Obstáculos y perspectivas al emplear tecnologías de información para enseñar contabilidad. Educación y humanismo, 21(37), 104-119. https://doi.org/10.17081/eduhum.21.37.3461

Martínez, J., y Lugo, L. (2018). Agentes que impulsan la capacidad innovativa en unidades universitarias de investigación agropecuaria: un análisis prospectivo. Omnia, 24(1), 87-99. http://produccioncientificaluz.org/index.php/omnia/article/view/32715

Martínez, N., y Rodríguez, A. (2018). Alfabetización y competencia digital en personas mayores: el caso del aula permanente de formación abierta de la Universidad de $\begin{array}{llll}\text { Granada } \quad \text { (España). Revista 39(10), } & \text { 37-62. }\end{array}$ https://www.revistaespacios.com/a18v39n10/a18v39n10p37.pdf

Ministerio de Educación Nacional (2013). Competencias TIC para el desarrollo profesional docente. Bogotá: Imprenta nacional.

Ministerio de Salud de la República de Colombia (2020). Colombia confirma su primer caso de COVID-19. https://www.minsalud.gov.co/Paginas/Colombia-confirma-su-primercaso-de-COVID-19.aspx

Moll, S. (2018). Los cinco pilares de la competencia digital docente y sus finalidades. https://www.educaciontrespuntocero.com/noticias/competencia-digital-docente/

Olmedo, J., Gómez, M., y Pintor, M. (2014). Estrategias innovadoras en el aula: implementación de un objeto virtual de aprendizaje. Educación y humanismo, 16(26),

58-72. http://revistas.unisimon.edu.co/index.php/educacion/article/view/2347

Osuna, S., y López, J. (2015). Modelo de evaluación educomunicativa en la educación $\begin{array}{llll}\text { virtual. Opción, 31(Especial 832-853. } & \text { 2), }\end{array}$ http://produccioncientificaluz.org/index.php/opcion/article/view/20419/20332

Pincay, J. (2018). Reflexiones sobre la accesibilidad web para el contenido educativo en los sistemas de administración de aprendizaje. Revista electrónica formación y

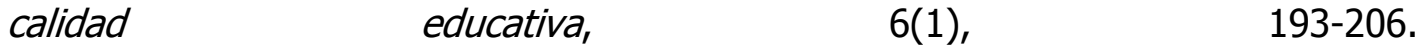
https://refcale.uleam.edu.ec/index.php/refcale/article/view/2553/1457 
Presidencia de la República (2020, 22 de marzo). Decreto número 457. https://dapre.presidencia.gov.co/normativa/normativa/DECRETO\%20457\%20DEL \%2022\%20DE\%20MARZO\%20DE\%202020.pdf

Semana (20 de abril de 2020). Duque anuncia que la cuarentena en Colombia se extenderá hasta el 11 de mayo. https://www.semana.com/nacion/articulo/cuarentena-en-colombia-se-extenderahasta-el-11-de-mayo-por-coronavirus/664820

Tamayo, M. (2004). El proceso de la investigación científica. Ciudad de México, México: Editorial Limusa.

Tarango, J., y Machado, Y. (2012). Diseño de acciones de alfabetización informacional en TIC para profesionales del sector de la salud en Cuba. Revista interamericana de bibliotecología, 35(2), 173-187. https://www.redalyc.org/articulo.oa?id=179026369005

Torres, N.; Pessoa, T., y Gallego, M. (2019). Intervención y evaluación con tecnologías de la competencia en seguridad digital. Digital education review, (35), 111-129. https://revistes.ub.edu/index.php/der/article/view/27399

Vargas, G. (2019). Competencias digitales y su integración con herramientas tecnológicas en educación superior. Cuadernos Hospital de clínicas, 60(1), 88-94. http://www.scielo.org.bo/scielo.php?script=sci_arttext\&pid=S165267762019000100013

Varguillas, C., y Bravo, P. (2020). Virtualidad como herramienta de apoyo a la presencialidad: análisis desde la mirada estudiantil. Revista de Ciencias sociales, XXVI(1), 219-232. http://produccioncientificaluz.org/index.php/rcs/article/view/31321/32371 